\title{
Human cortical representations for reaching: Mirror neurons for execution, observation, and imagery
}

\author{
Flavia Filimon, ${ }^{\mathrm{a}, *}$ Jonathan D. Nelson, ${ }^{\mathrm{a}, \mathrm{b}}$ Donald J. Hagler, ${ }^{\mathrm{a}}$ and Martin I. Sereno ${ }^{\mathrm{a}}$ \\ ${ }^{a}$ Department of Cognitive Science, University of California, San Diego, 9500 Gilman Dr. \#0515, La Jolla, CA 92093-0515, USA \\ ${ }^{\mathrm{b}}$ Computational Neurobiology Laboratory, Salk Institute, La Jolla, CA, USA
}

Received 5 April 2007; revised 27 May 2007; accepted 8 June 2007

Available online 18 June 2007

\begin{abstract}
We used functional magnetic resonance imaging (fMRI) to map the cortical representations of executed reaching, observed reaching, and imagined reaching in humans. Whereas previous studies have mostly examined hand actions related to grasping, hand-object interactions, or local finger movements, here we were interested in reaching only (i.e. the transport phase of the hand to a particular location in space), without grasping. We hypothesized that mirror neuron areas specific to reaching-related representations would be active in all three conditions. An overlap between executed, observed, and imagined reaching activations was found in dorsal premotor cortex as well as in the superior parietal lobe and the intraparietal sulcus, in accord with our hypothesis. Activations for observed reaching were more dorsal than activations typically reported in the literature for observation of handobject interactions (grasping). Our results suggest that the mirror neuron system is specific to the type of hand action performed, and that these fronto-parietal activations are a putative human homologue of the neural circuits underlying reaching in macaques. The parietal activations reported here for executed, imagined, and observed reaching are also consistent with previous functional imaging studies on planned reaching and delayed pointing movements, and extend the proposed localization of human reach-related brain areas to observation as well as imagery of reaching.
\end{abstract}

(C) 2007 Elsevier Inc. All rights reserved.

\section{Introduction}

Despite the long tradition of studying space perception from a purely visual perspective, recent research has revealed that motor actions are a key part of space perception. Interactions with the world, such as through eye and hand movements, contribute to a representation of space that is not just visual, but also motor. Mirror neurons for hand actions are one example of such visuomotor representations.

\footnotetext{
* Corresponding author. Fax: +1 8585341128.

E-mail address: ffilimon@cogsci.ucsd.edu (F. Filimon).

Available online on ScienceDirect (www.sciencedirect.com).
}

Electrophysiological studies in macaques have identified several frontal areas involved in hand action representations (Preuss et al., 1996; Muakkassa and Strick, 1979; Matelli and Luppino, 2001; Rizzolatti et al., 1988). For instance, both dorsal premotor cortex (PMd, or F2 and F7) and ventral premotor cortex (PMv, or F4 and F5) contain arm/hand representations that are specific to certain motor actions, such as grasping or reaching (Matelli and Luppino, 2001). More specifically, neurons that respond to both hand action execution (e.g. grasping) and hand action observation (e.g. observed grasping) have been found in macaque ventral premotor area F5 (for a review, see Rizzolatti and Craighero, 2004; Rizzolatti et al., 1996a; Buccino et al., 2004a). These "mirror neurons" suggest an observation-execution matching system that allows monkeys to recognize actions performed by other individuals by mapping them onto their own motor representations.

Mirror neurons have also been studied in macaque parietal cortex, in particular in the inferior parietal lobule (Fogassi et al., 1998). Their presence in the parietal lobe is in accord with the many hand/arm motor representations found in monkey parietal cortex, such as the anterior intraparietal area (AIP), which deals with grasping and object manipulation, the medial intraparietal area (MIP), which controls arm movements during reaching, and other arm-related parietal areas, such as areas V6A, 5, and 7 (Johnson et al., 1996; Stepniewska et al., 2005; Fattori et al., 2001; Galletti et al., 1997; Andersen and Buneo, 2002; Buneo et al., 2002; Ferraina et al., 2001; Kalaska, 1996; Battaglia-Mayer et al., 2000; Culham and Kanwisher, 2001). These parietal areas have specific premotor targets: distinct parieto-frontal neural circuits have been found in the macaque brain for grasping (AIP-F5), and reaching (MIP/V6A-F2vr), for instance (Matelli and Luppino, 2001).

Over the last few years, functional imaging studies in humans have begun to explore mirror neuron activations for hand actions in humans (for a review, see Grèzes and Decety, 2001; Decety and Grèzes, 1999; Buccino et al., 2004a). Investigation of the mirrorneuron system in humans has mainly focused on the involvement of ventral premotor cortex and the inferior frontal gyrus (Broca's area), an area thought to be the human homologue of macaque F5 (Rizzolatti et al., 1996a). 
Several human neuroimaging studies have investigated observation of grasping or of object manipulation (Grafton et al., 1996a; Binkofski et al., 1999; Buccino et al., 2001; Johnson-Frey et al., 2003; Molnar-Szakacs et al., 2006; Tai et al., 2004; Grèzes et al., 2003). Other studies have compared grasping observation with grasping execution or imitation (Rizzolatti et al., 1996b; Grèzes et al., 2003; Hamzei et al., 2003; see also a neuromagnetic study by Nishitani and Hari, 2000), observation versus imitation of simple finger movements (Iacoboni et al., 1999), or observation versus execution of more complex finger movements, such as playing guitar strings (Buccino et al., 2004b). In addition to execution and observation of hand actions, mental simulation (imagery) of hand actions, such as imagined grasping, has also been investigated (Grafton et al., 1996a; Grèzes and Decety, 2001). Finally, there is also a literature on observation of pantomimes and planning of tool use (Decety et al., 1997; Johnson-Frey et al., 2005) as well as execution of pantomimes (e.g. pantomimed grasping compared to actual grasping, and pantomimed reaching to touch an object compared to actual reaching to touch an object; Króliczak et al., 2007).

The majority of these studies involve hand-object interactions, whether through prehension, touching of an object, or preshaping of the hand during a pantomimed interaction with a missing object. In other cases precise, local, finger movement that is limited to the hand was studied, such as finger lifting (Iacoboni et al., 1999). A majority of these studies have found activations in ventral premotor/ inferior frontal cortex as well as in the inferior parietal lobe. The frontal activations included Broca's area, the putative human homologue of macaque F5, in most cases. In some cases, the superior parietal lobe, precuneus, and the intraparietal sulcus were also involved, with greater activations in the hemisphere contralateral to the moving hand. Typically studies that involve less of a transport phase in both observation and execution conditions (e.g. Rizzolatti et al., 1996b, where only the final phase of the hand grasping an object was viewed) find activations in the inferior frontal gyrus and the inferior parietal lobule, but not the superior parietal lobule. Tasks that involve a greater transport phase (e.g. Hamzei et al., 2003, where a cup was grasped and moved from the lap to the mouth; Grafton et al., 1996b; Culham et al., 2003) do activate the superior parietal lobule. It is unclear, however, why some studies report superior parietal activations and others do not.

Whereas a number of these studies have focused on hand actions that involve hand preshaping (e.g. during grasping or pantomimed grasping) or precise local hand movements (whether an object is present or not), less is known about which brain areas are involved in the execution, observation, and imagery of hand actions that do not involve object-directed movement, local finger configurations, or touching of an object. For instance, it is unknown if just the transport phase of the hand through space, such as during reaching, activates its own set of mirror neurons during execution, observation, and imagery of reaching. In other words, few studies have investigated whether mirror neuron activations change with hand actions in accordance to the fronto-parietal neural circuits identified in macaques, with $\mathrm{AIP}-\mathrm{F} 5$ representing grasping-related actions, and MIP-F2vr representing reaching-related actions. Are there mirror neuron activations for reaching (without grasping or touching) in humans, and how do they compare to mirror neuron activations for grasping, hand-object interactions, or smaller finger movements such as finger lifting? Reaching movements are different from the aforementioned hand actions in that only a transport phase of the hand is required, where preshaping of the hand for appropriate hand-object interactions or even touching is not necessary. For reaching, extraction of the visual properties of the object is not necessary; instead, the hand and arm need guided toward the appropriate point in space, regardless of what is located at that point in space. Buccino et al. (2001) found a somatotopic organization for observation of movements performed with different effectors (hand, mouth, or foot) in both premotor and parietal cortex. This suggests multiple mirror neuron systems in the human brain, dependent on the particular effector with which an action is performed. It remains unclear whether there is a similar systematic differentiation between mirror neuron activations for different types of hand movements, e.g. between reaching movements, and handobject interactions (including touching) or grasping movements. Observation of static images depicting object prehension versus observation of images depicting object touching results in an increase in activation in bilateral inferior frontal gyrus, suggesting that the less hand-object interaction (touching instead of grasping), the less involvement of the inferior frontal gyrus (Johnson-Frey et al., 2003). It remains unknown whether observation of reaching without touching of an object is similar to this static image observation task, in terms of not activating the inferior frontal gyrus. Likewise, it remains unknown which other brain areas, outside Broca's area or premotor cortex, are differentially active for reaching observation relative to grasping observation.

Reaching (defined as involving some arm transport as opposed to just hand/finger movements) has been difficult to investigate with fMRI, due to problems with head motion caused by the moving hand (see Culham et al., 2006). Typically, fMRI studies have investigated a proxy for reaching, such as delayed pointing (Medendorp et al., 2005; Hagler et al., 2007; Connolly et al., 2003; Astafiev et al., 2003; DeSouza et al., 2000) or using a joystick cursor to a visual target (e.g. Grefkes et al., 2004; Lacquaniti et al., 1997). Despite the difficulties, some fMRI studies have investigated reaching-to-point movements (Kawashima et al., 1996; Frey et al., 2005, Desmurget et al., 2001), reaching-to-touch (e.g. with the knuckles) (Culham et al., 2003), or reaching-to-grasp (Chapman et al., 2002; Frey et al., 2005). Prado et al. (2005) investigated actual reaching. The activations reported for these tasks typically include the medial IPS as well as the precuneus, i.e. more medial areas of the superior parietal lobe. Other activations include the parietooccipital cortex, supplementary motor cortex, and the cingulate sulcus. In addition, an area located at the junction between the anterior IPS and the inferior postcentral sulcus, hypothesized to be the human homologue of AIP (see Frey et al., 2005; Binkofski et al., 1999), is activated during both grasping and reach-to-point tasks as well as during some pointing tasks (Culham et al., 2006). This suggests some overlap between reaching and grasping. Both grasping and reaching activate the hemisphere contralateral to the moving hand substantially more than the ipsilateral hemisphere. In general, activations for reaching tasks tend to be more dorsal and medial in the parietal lobe (medial to the IPS) compared to object manipulation. Studies that involve a transport phase of the hand prior to an executed grasp (e.g. Culham et al., 2003; Grafton et al., 1996b) tend to find superior parietal activations, whereas studies in which the transport phase is not present, involving just local grasping (e.g. Rizzolatti et al., 1996b) tend to find more inferior parietal activations.

According to Culham et al. (2006), reaching-to-point tasks may actually involve preshaping the hand as well as calculating object properties such as the centroid of the shape, and thus may be different from reaching alone. Similarly, pointing may be expected to be different from actual reaching. Areas activated by delayed 
pointing activations in humans may thus not be exactly equivalent to macaque reach-related areas. It is also important to note that reaching-to-touch an object still represents a hand-object interaction, even if no grasping occurs, and that this task may thus be different from reaching without touching. Likewise, a grasping pantomime, or a pantomime in which an object is implied but not present, also represents an object-directed action: the object is implicit, and the pantomiming hand is preshaped accordingly. Since no preshaping of the hand needs to happen in reaching-only, pantomimed hand-object interactions are still likely to differ from reaching by activating grasping-related neural circuits.

While several studies have investigated the execution aspect of grasping or reaching-like hand actions, we are aware of no studies that have investigated parieto-frontal mirror neurons for reaching per se. It is thus unknown which brain areas are involved in both execution of reaching and in observation as well as mental simulation of reaching.

In addition, the majority of previous mirror neuron studies have compared only two types of conditions at a time, i.e. either execution with observation of hand actions, or observation with imagery, or execution/imitation with imagery of hand actions (see Grèzes and Decety, 2001). Since different studies used different hand actions (e.g. object manipulation or finger tapping) as well as different objects, it is difficult to draw general conclusions about which brain areas are involved in all three conditions (action execution, observation, and imagery) for a particular hand action. It is also unclear whether there are differences in activation levels between observation, imagery, and execution within mirror neuron areas-for instance, whether observing a hand action is more potent at driving mirror neurons than imagining the same hand action.

Here, we compare execution of visually-guided reaching (i.e. reaching directly towards visually presented targets without the use of a mirror) with observed as well as imagined reaching in the same experiment. Since our task involves no explicit touching or grasping of objects, and therefore no preshaping of the hand to match a viewed object shape, mirror neuron activations for observation as well as imagery of reaching should involve more of the reachrelated substrates than hand manipulation-related substrates.

\section{Methods}

\section{Participants}

Sixteen subjects (ten males) participated in this experiment (age range 19-48). One subject was discarded due to excessive head motion. All subjects were right-handed and had normal or corrected-to-normal vision. Human subjects' approval was obtained from the UCSD Institutional Review Board. All subjects gave informed consent.

\section{Stimuli}

The stimuli used were photographs of abstract wooden shapes on a black background (see Figs. 1a and c), with five different views taken per shape. The abstract shapes served as targets for the reach, imagined reach, or passive viewing. We chose abstract shapes in order to 1) prevent object recognition/verbalizing, as in the case of common objects such as pens, cups, bananas, etc., and 2) to motivate the reach, since naturalistic reaching usually occurs towards objects rather than simple dots in space. In all photographs, lighting
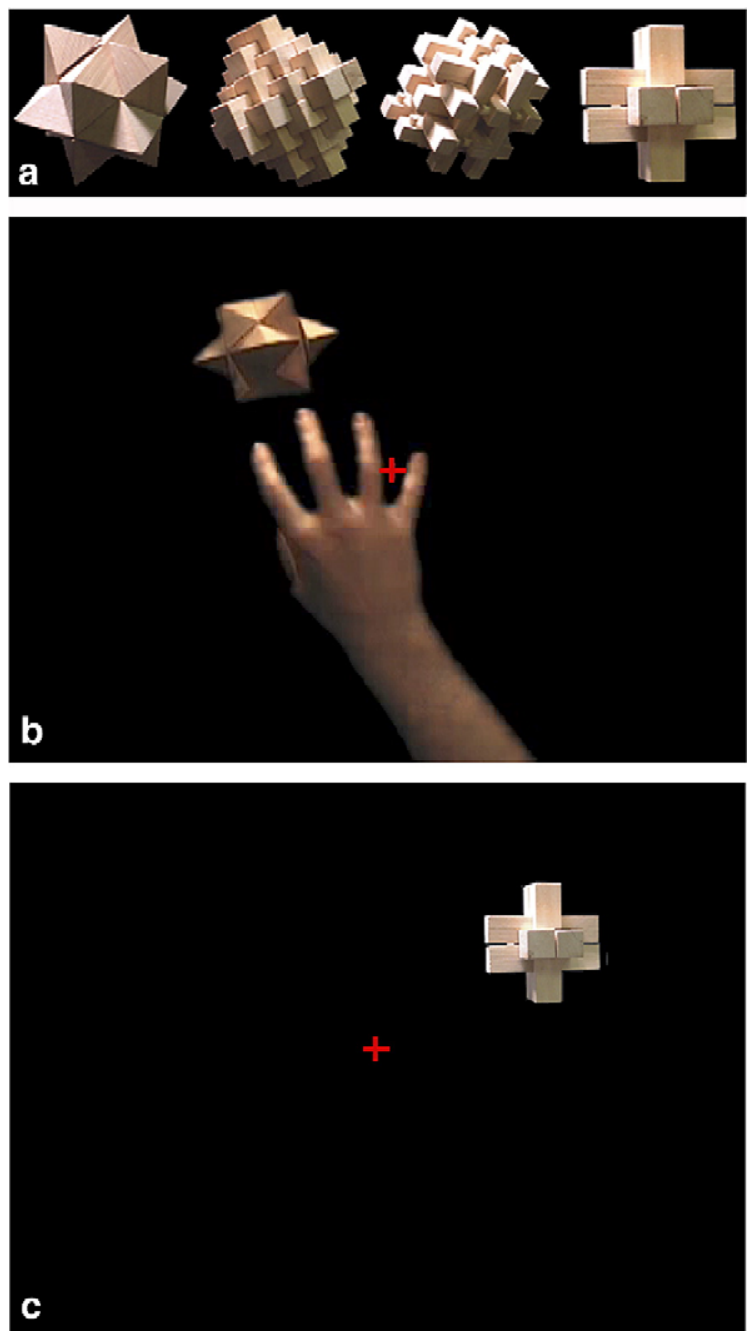

Fig. 1. Stimuli used in the experiment. (a) The four shapes used as visual stimuli. Each shape was photographed from five different angles, yielding a total of 20 stimulus images. (b) Still frame from the observation of reaching video, used in the observed reaching condition. A human hand is seen reaching towards one of the shapes. (c) Example trial during either executed or imagined reaching, or passive viewing of objects (baseline). The subject's task was indicated beforehand with a message.

was held constant (always from the top left), thus giving the objects a three-dimensional appearance.

In addition, video clips of an actor's right hand reaching towards the same abstract wooden shapes (see Fig. 1b) were recorded with a digital camera and edited in Final Cut Pro (version 3.9). The video clips served as stimuli in the observed reaching condition. The hand was seen slowly reaching towards the objects on a black background, with the final hand position being just before the grasp. Only the forearm appeared in all frames. Lighting was as in the photographs. The objects in the video clips used in the observed reaching condition were slightly smaller than the objects presented during the reaching, imagined reaching, and fixation conditions. The video hand was likewise somewhat smaller than subjects' actual hand, so that as much of the transport phase of the reach was observable as possible on a small screen.

Stimuli were projected on a screen inside the scanner $\sim 12$ in. in front of the subject's chin, above their torso, such that the subject 
could directly view the screen without a mirror and reach directly towards the display without touching the screen. A standard video projector with a 7.38-12.3" focal length Xtra Bright Zoom replacement lens (Buhl Optical, USA) was used to display stimuli running on a PowerBook (500 MHz G3 running Mac OS X) at SVGA resolution. The screen was attached with velcro to the ceiling of the scanner bore. Subjects' heads were tilted forward towards the screen. This ensured that the reaching movement was as far as possible from the subject's head to prevent a disturbance of the magnetic field around the head. In addition, the tilted head position allowed for direct, i.e. visually guided, reaching. The starting hand position was the center of the subject's chest.

The object images were presented at constant eccentricity $\left(\sim 10^{\circ}\right)$ from the central fixation cross at a random polar angle. One of the five views of one of the four objects was selected randomly and displayed around the fixation cross. Objects subtended a visual angle of $\sim 6^{\circ}$, while the entire display for all conditions subtended a visual angle of $\sim 35^{\circ}$. Each object was displayed for $4 \mathrm{~s}$, giving subjects enough time to slowly reach and retract their hand, after which the next shape appeared immediately. Subjects were instructed to reach slowly with their open right hand towards the object images without touching the screen and without closing the hand. Movement of the elbow was minimized as it was supported and partially immobilized by foam pads, i.e. subjects mainly moved their forearm. Subjects maintained fixation during the reach and throughout the experiment. To prevent head motion, a custom-made bite-bar was used, consisting of each subject's individual dental impression attached to an adjustable 4-ball-joint yoke. Additional foam pads around the head were used to stabilize the head. Prior to scanning, subjects were trained to reach slowly in a practice session outside the scanner. In addition, their eye movements were monitored during these practice reaching trials, to ensure they were able to reach without visually tracking their hand or saccading to the targets. All subjects were able to reach accurately while maintaining fixation on the fixation cross in the center of the screen. Additional eye tracker data from one subject collected outside the scanner showed that central fixation was maintained during execution, observation, and imagery of reaching, as well as during baseline passive viewing of objects.

\section{Experimental design}

A pseudo-random block design was used. Subjects participated in three experimental conditions and one control condition: 1) actual reaching towards images of objects, 2) observation of reaching towards the same objects, 3) imagined reaching to the visually displayed objects, and 4) fixation with passive viewing of the objects. In 1), 3) and 4) the images of wooden shapes described above were presented randomly around the fixation cross, at fixed eccentricity and random angle, for $4 \mathrm{~s}$ each. In the observed reaching condition (2), subjects viewed video clips of a human right hand reaching towards the shapes, while fixating on the central fixation cross, without moving their hand. The object locations and thus possible reach directions in the video clips were randomized and matched the locations of the shapes in the other conditions. For imagined reaching (3), subjects imagined their right hand moving towards the objects at the same speed as their actual reach, while fixating on the central fixation cross. The hand was held stationary on the chest throughout this condition.

A 2-s message (e.g. "Observe") at the beginning of each block of trials indicated to the subject which condition was about to begin.
Each condition was presented for $32 \mathrm{~s}$ at a time (block length= $32 \mathrm{~s}$ ), with each condition being repeated 4 times during an $8 \mathrm{~min}$ $32 \mathrm{~s}$ run. The order of conditions was pseudo-randomized. Each subject participated in a minimum of two $8 \mathrm{~min} 32 \mathrm{~s}$ runs.

\section{Image acquisition}

Magnetic resonance images were collected with a Varian 3T MRI scanner using an end-capped $23 \mathrm{~cm}$ quadrature head coil designed and built by Eric Wong (University of California, San Diego, Center for fMRI). Functional scans were collected using an echo-planar T2*-weighted gradient echo pulse sequence (28 contiguous axial slices, $3.75 \times 3.75 \times 3.8 \mathrm{~mm}$ voxel size, $64 \times 64$ matrix, $\mathrm{TR}=2000 \mathrm{~ms}, 258$ repetitions per scan, $\mathrm{TE}=27.4 \mathrm{~ms}$, flip angle $=90^{\circ}$, bandwidth $=1950 \mathrm{~Hz} /$ pixel). The slice volume included the entire brain except for the inferior tip of the temporal lobes. Stimulus presentation began after the first 2 TRs, which were discarded, to allow the magnetization to reach a steady state. A T1weighted MPRAGE alignment scan $(1 \times 1 \times 2 \mathrm{~mm}, \mathrm{TR}=16 \mathrm{~ms}$, $\mathrm{TE}=6.6 \mathrm{~ms}$, flip angle $=18^{\circ}, 256 \times 200$ matrix $)$ was collected in the same scanning session to align the functional images to a previously obtained high-resolution $(1 \times 1 \times 1 \mathrm{~mm})$ T1-weighted MPRAGE scan collected on a $1.5 \mathrm{~T}$ Siemens or $3 \mathrm{~T}$ Varian scanner.

\section{Data processing and statistical analysis}

Each subject's cortical surface was reconstructed from the highresolution $(1 \times 1 \times 1 \mathrm{~mm})$ T1-weighted MPRAGE anatomical scan using FreeSurfer (Fischl et al., 1999a; Dale et al., 1999). The functional images were superimposed on the alignment scan (MPRAGE, $1 \times 1 \times 2 \mathrm{~mm}$ resolution) and then registered with the high-resolution $(1 \times 1 \times 1 \mathrm{~mm})$ anatomical image, allowing for functional data to be registered with and painted onto each subject's reconstructed cortical surface. Statistical analyses were carried out using Analysis of Functional Neuroimages (AFNI) (Cox, 1996).

\section{Motion correction}

The functional runs were concatenated, yielding at least 512 TRs per subject, and were corrected for motion artifacts and linear drift using AFNI's volreg (volume registration) function, in which all functional images were registered to the middle of the second functional run. Six estimated motion (3 rotation and 3 translation) parameters were obtained during this registration.

\section{Deconvolution analysis}

Each individual subject's blood-oxygenation-level-dependent (BOLD) activations were analyzed using AFNI's 3dDeconvolve (Cox, 1996; Ward, 2000a) general linear model. Motion parameters obtained during volume registration were added to the baseline model as additional regressors. We used a quadratic polynomial to fit and remove the baseline. Hemodynamic responses were modeled at four different lag times (1, 2, 3, and 4 TRs). 3dDeconvolve uses multiple regression to estimate the goodness of fit between estimated system impulse response functions and the actual fMRI time series data, for each voxel. Program 3dDeconvolve does not assume a specific shape of the hemodynamic response function (HRF) (e.g. a fixed waveform such as a square wave), but instead estimates the shape of the waveform voxel by voxel, based on the data itself, using a sum of scaled and time-delayed versions of the stimulus time series to model the system response. Thus the shape and amplitude of the HRF was allowed to vary at each of the 4 lag points. 
Correlation coefficients and $F$-statistics were generated for the area under the hemodynamic response function. Six general linear tests were carried out: observed, imagined, and actual reaching each versus baseline, as well as pairwise comparisons (reach versus observe or imagine, observe versus imagine).

\section{Group analysis}

The group data were analyzed using cortical surface-based averaging algorithms in FreeSurfer (Fischl et al., 1999b). Each subject's reconstructed cortical surface was sphered and morphed to an average spherical surface of 40 subjects using a best-fit sulcus alignment. Individual functional activations and statistics could then be interpolated onto the average sphere and averaged across subjects.

Surface-based smoothing using 16 smoothing steps was performed before resampling coefficients onto the average spherical surface, which corresponds to a full-width, half-max (FWHM) Gaussian filter of approximately $4.9 \mathrm{~mm}$ (Hagler et al., 2006). The average activations and $F$-stats were then resampled back onto a single subject's inflated cortical surface for display purposes using FreeSurfer. Surface-based averaging across subjects has been shown to yield a better alignment of activations across subjects, leading to less blurring and less loss of signal compared to Talairach methods (Fischl et al., 1999b).

$F$-statistics and regression coefficients (means) for the group analysis were generated for each condition (observed, imagined, and executed reaching, versus baseline) as well as for pairwise comparisons (executed versus observed reaching, executed versus imagined reaching, and observed versus imagined reaching) using AFNI's mixed-effects, two-factor 3dANOVA2 with repeated measures (within-subject). Condition was treated as a fixed effect with three levels while subject was a random effect.

\section{Clustering}

To correct for multiple comparisons, surface-based cluster-size exclusion (Hagler et al., 2006) was used for surface-based groupaveraged data, with $t$-statistics thresholded at $p<0.05, p<0.01$, $p<0.005$, and $p<0.001$, corresponding to cortical surface clusters of $352,148,112$, and 60 contiguous vertices, respectively. Clusters with fewer contiguous vertices than the specified cluster sizes were excluded. AFNI's AlphaSim (Ward, 2000b) was adapted to cortical surface data to ensure that the corrected $p$-value for these thresholds was 0.05 (Hagler et al., 2006).

\section{ROI analysis}

Regions of interest (ROIs) were selected according to the following criteria: 1) activated voxels overlapped for observed, imagined, and executed reaching (all three conditions); 2) the overlapping activations had to survive a threshold of $p<0.001$ in each of the three conditions, relative to baseline; and 3) voxels were in anatomical regions identified in the literature as being involved in action production as well as observation, namely, premotor and parietal cortices. Two regions emerged that fulfilled each of these criteria: one in dorsal premotor cortex (superior frontal gyrus/sulcus) and one in posterior parietal cortex. The ROIs were saved as surface patches in FreeSurfer, with MATLAB scripts extracting the overlap between the three conditions. Each voxel's time series was normalized by its mean intensity and then averaged together with all voxels within each ROI using MATLAB. Each ROI's normalized time series was then analyzed with AFNI's input -1D 3dDeconvolve option to extract percent signal change coefficients for each condition.

\section{Results}

All three conditions (executed, observed, and imagined reaching) activated a fronto-parietal network when compared to baseline (Fig. 2, Tables 1-3). Fig. 2 shows average group activations displayed on an inflated cortical surface. The activations for all three conditions overlapped in dorsal premotor cortex, as well as in superior parietal cortex and in the intraparietal sulcus (see Figs. 4a and b). Fig. 3 shows activations from 5 individual subjects who are representative of the group of 15 subjects. Note that we are following the terminology used in Duvernoy's (1999) human brain atlas when using the term "dorsal premotor", which is defined as dorsal cortical areas anterior to the precentral gyrus, i.e. in this case the superior frontal gyrus and sulcus.

\section{Execution of reaching versus passive viewing of objects}

Visually-guided reaching involves motor, visual, as well as somatosensory components, and was therefore expected to activate several brain areas subserving these functions. Indeed, activations $(p<0.005$, corrected) were observed in primary motor and somatosensory cortex, dorsal premotor cortex (superior frontal gyrus and sulcus, extending onto the caudal aspect of the middle frontal gyrus), the inferior frontal gyrus, the supplementary motor area (medial aspect of the superior frontal gyrus), cingulate cortex, the posterior end of the Sylvian fissure, as well as superior and inferior parietal (supramarginal) cortex, the intraparietal sulcus (IPS), and the precuneus. Visual areas including the cuneus, lingual gyrus, superior occipital gyrus, middle occipital gyrus $(\mathrm{MT}+)$, the calcarine sulcus, and the posterior end of the superior temporal sulcus (STS) were also activated (see Fig. 2 and Table 1). Most activations were lateralized to or stronger in the left hemisphere, as expected for normal right-handed subjects moving their right hand only. For instance, primary sensorimotor and inferior frontal gyrus activation was present only in the left hemisphere, and dorsal premotor and superior parietal activations were much weaker in the right hemisphere compared to the left hemisphere. Interestingly, ventral premotor and inferior frontal gyrus activations, overlapping with Broca's area, were weaker compared to the dorsal premotor activations (superior frontal gyrus), consistent with the fact that our task involved reaching and no grasping.

\section{Observed reaching versus passive viewing of objects}

Although observed reaching consisted of simply viewing video clips of a reaching right hand, we expected to find activations not just in visual areas, but also in visuo-motor areas. In particular, we expected activations in premotor and parietal areas, in which mirror neurons have previously been found for observation of hand actions, as well as in areas that represent reaching. As expected, observation of reaching activated the intraparietal sulcus, the superior parietal lobule and precuneus, as well as dorsal premotor cortex (superior frontal gyrus/sulcus) ( $p<0.005$, corrected; Fig. 2, Table 2). In addition, observed reaching activated visual areas (superior occipital gyrus, cuneus, middle occipital gyrus), and the posterior end of the superior temporal sulcus. Activations were again stronger in the left hemisphere, with dorsal premotor activations strongly left-lateralized. Notably, no activations were observed in the inferior frontal gyrus (even at $p<0.05$, corrected), which may be due to the fact that there were no interactions between the observed hand and the objects in the videos. This is consistent 


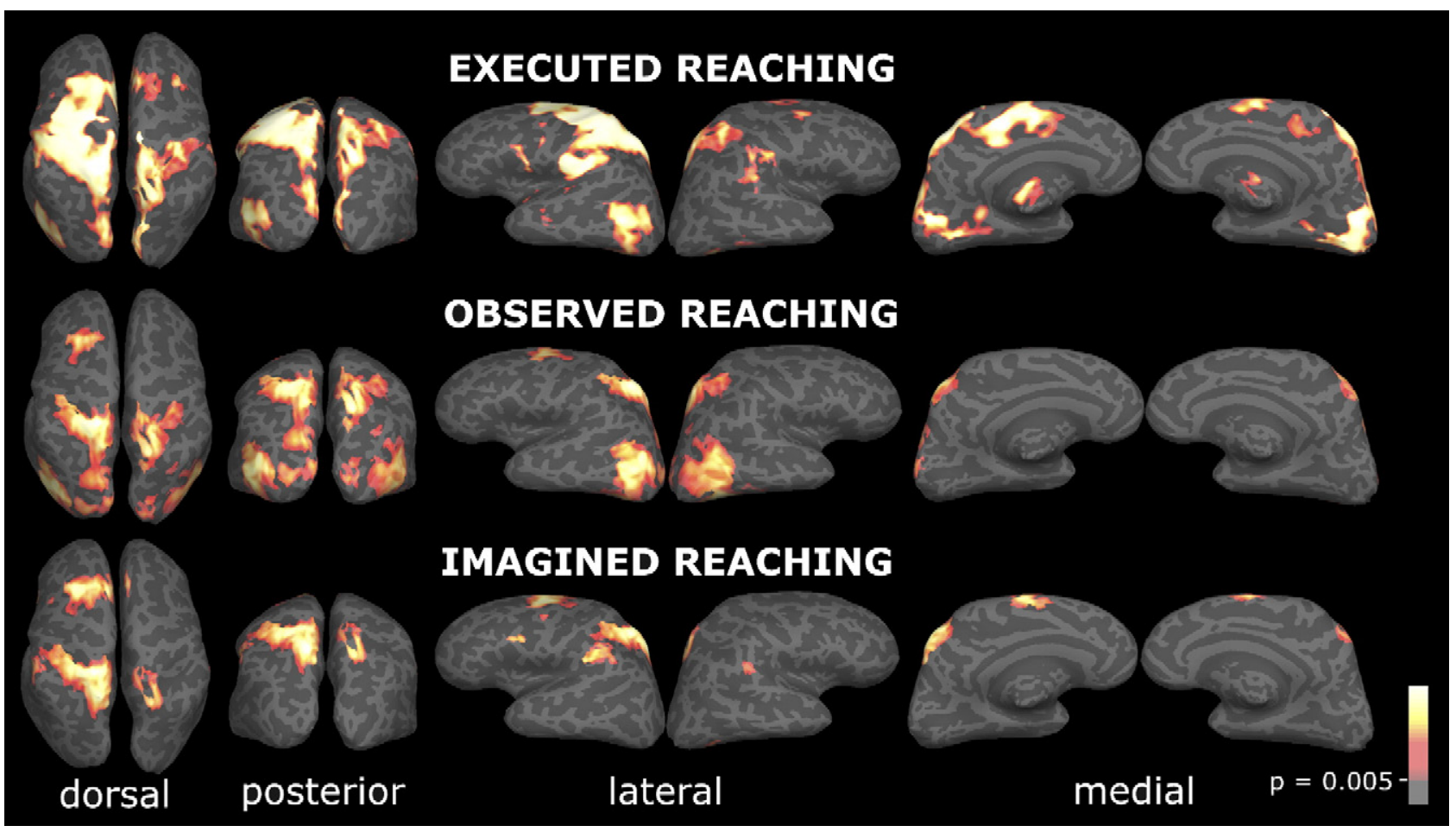

Fig. 2. Group surface-averaged activations for executed, observed, and imagined reaching, versus baseline, from 15 subjects, interpolated onto a single subject's inflated cortical hemispheres, viewed here from dorsal, posterior, lateral, and medial views. All activations displayed are significant at $p<0.005$ (corrected). Note the overlap in activations in superior parietal and dorsal premotor cortex (superior frontal gyrus and sulcus), for all three conditions, suggesting the presence of mirror neurons in those areas.

with our hypothesis that ventral premotor/inferior frontal cortex is activated primarily during observation of hand-object interactions as well as hand configurations, rather than during observation of the transport phase of the hand.

\section{Imagined reaching versus passive viewing of objects}

Similar to observed reaching, imagined reaching activated the superior parietal lobule and intraparietal sulcus, the precuneus, as well as dorsal premotor cortex (superior frontal gyrus/sulcus) (see Fig. 2, Table 3). In addition, the inferior parietal lobule (supramarginal gyrus), the posterior end of the Sylvian fissure, and supplementary motor cortex (SMA) were activated, as was the inferior frontal gyrus (Broca's area). The activations $(p<0.005$, corrected) were left-lateralized with the exception of weak superior parietal, supramarginal, and supplementary motor area activations in the right hemisphere. The left-lateralization of imagined reaching activations suggests that imagery of hand movements engages a similar system as actual hand movements, which are also leftlateralized in the human brain. At a lower threshold $(p=0.05$, corrected), a small activation focus emerged within the left anterior occipital sulcus, overlapping with the lateral occipital activations for execution and observation of reaching. Other than this weak anterior occipital sulcus activation at $p=0.05$, no other occipital areas were activated during imagined reaching. This suggests that motor imagery is different from visual imagery, which activates visual areas. Note that since our baseline involved passive viewing of objects, any visual object-related activation unrelated to motor imagery was subtracted out. Although it has been suggested that the STS contains mirror neurons for hand movements (Perrett et al.,
1989), the STS was not activated during imagined reaching, suggesting that this area may respond to visually perceived biological motion, but not to imagined motion.

\section{Overlap between executed, observed, and imagined reaching}

All three conditions activated two main foci (Fig. 4, Table 4): a frontal region in the left dorsal premotor cortex (superior frontal gyrus and sulcus) and a parietal region in the left medial intraparietal sulcus and in the left superior parietal cortex (including the superior parietal gyrus and transverse parietal sulcus). Activations also overlapped in the right superior parietal cortex, although they were much weaker and the overlapping focus was much smaller. The superior parietal overlap in the left hemisphere extended medially into the superior aspect of the precuneus, in an area located anterior to the parieto-occipital sulcus and posterior to the cingulate sulcus (see Fig. 4b). In addition, activations for executed and imagined reaching (but not for observed reaching) overlapped in the left and right medial superior frontal gyrus (SMA), the left and right inferior parietal lobule (supramarginal gyrus), the posterior end of the Sylvian fissure, and the left inferior frontal gyrus. Consistent with previous findings (Grèzes and Decety, 2001), executed reaching activations in the SMA extended more caudally than imagined reaching SMA activations, although there was some overlap between the two. Conversely, activations for executed and observed reaching (but not for imagined reaching) overlapped in the left middle occipital gyrus and surrounding sulci $(\mathrm{MT}+)$. This suggests that imagined reaching is more potent than observed reaching at driving motor preparation areas such as supplementary motor cortex. In contrast, observation of reaching drives visual motion areas 
Table 1

Average MNI-space coordinates (in $\mathrm{mm}$ ) for activations during execution of reaching versus baseline

\begin{tabular}{|c|c|c|c|c|c|c|c|c|}
\hline \multirow[t]{2}{*}{ Brain area } & \multicolumn{4}{|l|}{ Left } & \multicolumn{4}{|c|}{ Right } \\
\hline & $x$ & $y$ & $z$ & $\begin{array}{l}\text { No. of } \\
\text { subjects }\end{array}$ & $x$ & $y$ & $z$ & $\begin{array}{l}\text { No. of } \\
\text { subjects }\end{array}$ \\
\hline $\begin{array}{l}\text { Lateral fissure, } \\
\text { posterior segment }\end{array}$ & -45 & -41 & 26 & 14 & 54 & -35 & 26 & 13 \\
\hline $\begin{array}{l}\text { Superior precentral } \\
\text { sulcus }\end{array}$ & -23 & -14 & 59 & 14 & & & & \\
\hline Precentral gyrus & -33 & -18 & 70 & 14 & 37 & -11 & 64 & 13 \\
\hline Postcentral gyrus & -37 & -30 & 69 & 14 & 34 & -38 & 69 & 7 \\
\hline Central sulcus & -31 & -28 & 52 & 14 & & & & \\
\hline $\begin{array}{l}\text { Superior postcentral } \\
\text { sulcus }\end{array}$ & -30 & -37 & 54 & 14 & 29 & -40 & 53 & 11 \\
\hline $\begin{array}{l}\text { Inferior frontal gyrus, } \\
\text { pars opercularis }\end{array}$ & -52 & 12 & 15 & 13 & & & & \\
\hline $\begin{array}{l}\text { Superior frontal } \\
\text { gyrus/sulcus }\end{array}$ & -21 & -2 & 63 & 14 & 23 & -5 & 67 & 12 \\
\hline Middle frontal gyrus & -31 & 3 & 64 & 6 & & & & \\
\hline $\begin{array}{l}\text { Superior temporal } \\
\text { sulcus }\end{array}$ & -48 & -47 & 5 & 9 & & & & \\
\hline Intraparietal sulcus & -33 & -46 & 46 & 14 & 26 & -52 & 49 & 12 \\
\hline Superior parietal gyrus & -27 & -55 & 65 & 14 & 20 & -59 & 65 & 14 \\
\hline Supramarginal gyrus & -57 & -38 & 46 & 14 & 60 & -31 & 42 & 14 \\
\hline Precuneus & -4 & -60 & 57 & 11 & 7 & -64 & 58 & 12 \\
\hline Cingulate sulcus & -10 & 0 & 41 & 13 & & & & \\
\hline $\begin{array}{l}\text { Supplementary motor } \\
\text { area (SMA) }\end{array}$ & -5 & -5 & 59 & 14 & 7 & 2 & 56 & 14 \\
\hline $\begin{array}{l}\text { Superior occipital } \\
\text { gyrus }\end{array}$ & -14 & -88 & 32 & 14 & 15 & -90 & 32 & 14 \\
\hline $\begin{array}{l}\text { Middle occipital } \\
\text { gyrus/anterior } \\
\text { occipital sulcus }\end{array}$ & -45 & -72 & 8 & 14 & & & & \\
\hline Lingual gyrus & -3 & -74 & -1 & 14 & 6 & -76 & -3 & 13 \\
\hline Cuneus & -2 & -88 & 9 & 14 & 5 & -86 & 17 & 13 \\
\hline Calcarine sulcus & -11 & -81 & 4 & 13 & 13 & -79 & 7 & 13 \\
\hline
\end{tabular}

One subject's MNI coordinates were anomalous ( $>30 \mathrm{~mm}$ different) and were thus excluded from the average of all MNI coordinates. All activations were at $p<0.005$ (corrected).

Table 2

Average MNI-space coordinates (in $\mathrm{mm}$ ) for activations during observed reaching versus baseline

\begin{tabular}{|c|c|c|c|c|c|c|c|c|}
\hline \multirow[t]{2}{*}{ Brain area } & \multicolumn{4}{|l|}{ Left } & \multicolumn{4}{|c|}{ Right } \\
\hline & $x$ & $y$ & $z$ & $\begin{array}{l}\text { No. of } \\
\text { subjects }\end{array}$ & $x$ & $y$ & $z$ & $\begin{array}{l}\text { No. of } \\
\text { subjects }\end{array}$ \\
\hline $\begin{array}{l}\text { Superior occipital } \\
\text { gyrus }\end{array}$ & -14 & -92 & 26 & 13 & 20 & -91 & 26 & 14 \\
\hline Intraparietal sulcus & -29 & -52 & 47 & 13 & 28 & -52 & 49 & 13 \\
\hline $\begin{array}{l}\text { Superior parietal } \\
\text { gyrus }(\mathrm{P} 1)\end{array}$ & -29 & -57 & 64 & 14 & 21 & -62 & 66 & 13 \\
\hline $\begin{array}{c}\text { Superior frontal } \\
\text { gyrus/sulcus }\end{array}$ & -23 & -6 & 60 & 14 & & & & \\
\hline $\begin{array}{l}\text { Middle occipital } \\
\text { gyrus/sulcus }\end{array}$ & -45 & -76 & 3 & 14 & 48 & -72 & 4 & 14 \\
\hline $\begin{array}{l}\text { Superior temporal } \\
\text { sulcus, caudal }\end{array}$ & -45 & -58 & 12 & 8 & 51 & -50 & 11 & 10 \\
\hline Precuneus & -6 & -62 & 52 & 11 & 7 & -65 & 52 & 11 \\
\hline Cuneus & -1 & -89 & 10 & 12 & & & & \\
\hline
\end{tabular}

All activations were at $p<0.005$ (corrected).
Table 3

Average MNI-space coordinates (in $\mathrm{mm}$ ) for activations during imagery of reaching versus baseline

\begin{tabular}{|c|c|c|c|c|c|c|c|c|}
\hline \multirow[t]{2}{*}{ Brain area } & \multicolumn{4}{|l|}{ Left } & \multicolumn{4}{|c|}{ Right } \\
\hline & $x$ & $y$ & $z$ & $\begin{array}{l}\text { No. of } \\
\text { subjects }\end{array}$ & $x$ & $y$ & $z$ & $\begin{array}{l}\text { No. of } \\
\text { subjects }\end{array}$ \\
\hline $\begin{array}{l}\text { Superior parietal } \\
\text { gyrus (P1) }\end{array}$ & -22 & -65 & 62 & 14 & 19 & -66 & 61 & 13 \\
\hline Intraparietal sulcus & -29 & -51 & 45 & 14 & & & & \\
\hline $\begin{array}{l}\text { Superior postcentral } \\
\text { sulcus }\end{array}$ & -28 & -40 & 55 & 10 & & & & \\
\hline Precuneus & -6 & -63 & 57 & 12 & & & & \\
\hline $\begin{array}{l}\text { Inferior parietal lobule: } \\
\text { supramarginal gyrus }\end{array}$ & -59 & -37 & 41 & 12 & 59 & -36 & 43 & 10 \\
\hline $\begin{array}{l}\text { Lateral fissure, } \\
\text { posterior segment }\end{array}$ & -48 & -44 & 26 & 13 & & & & \\
\hline $\begin{array}{l}\text { Superior frontal gyrus/ } \\
\text { sulcus }\end{array}$ & -22 & -3 & 61 & 14 & & & & \\
\hline $\begin{array}{l}\text { Superior precentral } \\
\text { sulcus }\end{array}$ & -26 & -12 & 55 & 14 & & & & \\
\hline $\begin{array}{l}\text { Inferior frontal gyrus, } \\
\text { pars opercularis }\end{array}$ & -50 & 16 & 15 & 11 & & & & \\
\hline $\begin{array}{l}\text { Supplementary Motor } \\
\text { Area (SMA) }\end{array}$ & -5 & -1 & 59 & 14 & 8 & 6 & 59 & 14 \\
\hline
\end{tabular}

All activations were at $p<0.005$ (corrected).

$(\mathrm{MT}+)$ more strongly than imagined reaching. In addition to $\mathrm{MT}+$, the activation in the middle occipital gyrus and surrounding sulci may include the extrastriate body area, which has been shown to respond to limb movements in the absence of visual input as well as to images of body parts (Astafiev et al., 2004). Astafiev et al. also found that imagery of pointing movements activates the extrastriate body area, although more weakly than actual movement. Our weaker activation ( $p=0.05$, corrected) for imagined reaching in lateral occipital cortex is consistent with Astafiev et al.'s findings.

We calculated the percent signal change for voxels $(p<0.001)$ within the two regions of interest (ROIs) defined by overlapping activations in dorsal premotor and parietal cortex shown in Figs. 4a and b. Fig. 5 shows the percent signal change and time course of activation for executed, imagined, and observed, reaching. A oneway ANOVA revealed significant differences between percent signal change means for executed, observed, and imagined reaching $(p=0.002)$ in the parietal ROI. To identify which conditions were different from each other on a pairwise basis, both two-tailed paired $t$-tests, and bootstrap resampling were used. In the parietal ROI, Reach was greater than Observe $(t(14)=3.25, p=0.0059$; bootstrap $p=0.0001)$, and Reach was greater than Imagine $(t(14)=3.94$, $p=0.0015$; bootstrap $p<0.0001)$. Observe and Imagine were not noticeably different from each other $(t(14)=0.07$, n.s.; bootstrap n.s.). In the premotor ROI, the trend was also for Reach to be greater than Observe $(t(14)=2.58, p=0.0217$; bootstrap $p=0.0041)$, and for Reach to be greater than Imagine $(t(14)=2.16, p=0.0490$; bootstrap $p=0.0170)$. As in the parietal ROI, Observe and Imagine were not significantly different $(t(14)=1.77$, n.s.; bootstrap n.s.). Both the $t$-test and bootstrap $p$ values suggest that in the premotor ROI, the differences between Reach and the other conditions were less strong than in the parietal ROI.

It is possible that execution of reaching drives mirror neuron areas more strongly than observation and imagery of reaching, or that other neurons, in addition to mirror neurons, are active during movement execution but not during observation and imagery. 


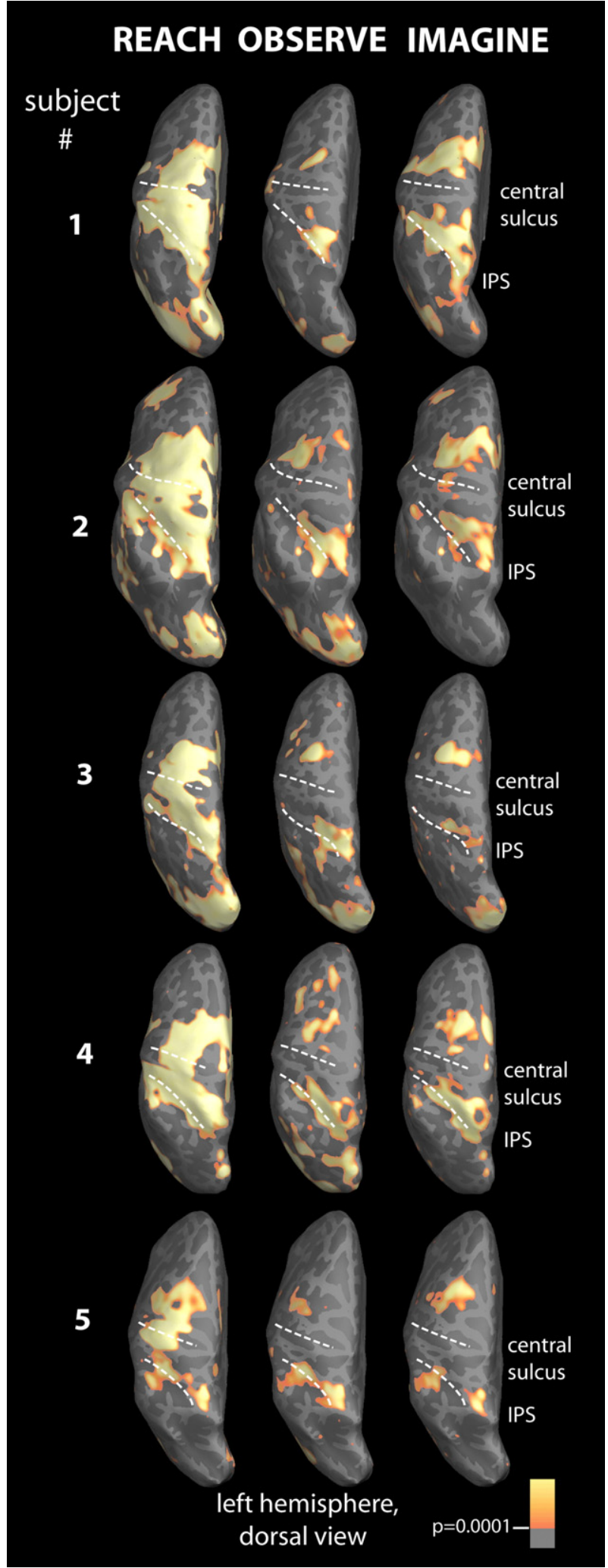

Fig. 3. BOLD activations for executed, observed, and imagined reaching, versus baseline, from five representative subjects. Activations are displayed on the left inflated hemisphere of each subject. Each subject's activations are significant at $p<0.0001$. Note that, despite inter-subject variability, each subject shows overlap in activations between all three conditions in dorsal premotor and superior parietal cortex. IPS=intraparietal sulcus.
Although fMRI cannot distinguish between these two possibilities, the fact that single unit recordings in macaques have found bimodal neurons with both visual and motor properties in premotor and parietal areas suggests that similar bimodal neurons may be involved in humans too, rather than separate populations of neurons.

\section{Execution versus observation of reaching}

Compared to observed reaching, executed reaching (Fig. 6) activated primary motor and somatosensory cortices, superior parietal cortex, the medial IPS, parts of dorsal premotor cortex (superior frontal gyrus/sulcus), the supplementary motor area, inferior parietal cortex (supramarginal gyrus), inferior frontal gyrus, the cingulate sulcus, cuneus, and lingual gyrus more strongly ( $p<0.005$, corrected). These differences were left-dominant. Conversely, compared to execution of reaching, observation of reaching activated the precuneus, the caudal intraparietal sulcus, the superior occipital gyrus, and the angular gyrus more strongly in both hemispheres $(p<0.005$, corrected). Note that neither reaching versus baseline nor observed reaching compared to baseline activated the angular gyrus significantly. Hence the decreased activation in this part of the inferior parietal lobule obtained in the reaching versus observed reaching contrast may be due to a decrease of activation in that region during reaching versus baseline, rather than greater activation for observed reaching compared to executed reaching.

\section{Execution versus imagery of reaching}

Compared to imagined reaching, executed reaching activated primary motor and somatosensory cortices as well as the superior parietal lobule and the medial IPS more strongly $(p<0.005$, corrected) (Fig. 6). In addition, the supramarginal gyrus, the middle occipital gyrus $(\mathrm{MT}+)$, a small part of dorsal premotor cortex (superior frontal gyrus), the cuneus, and the lingual gyrus were also activated more strongly during executed reaching than imagined reaching. Conversely, imagined reaching activated the caudal intraparietal sulcus, the angular gyrus bilaterally, and the precuneus more strongly than actual reaching. As in the executed reaching versus observed reaching contrast, the angular gyrus was not actually significantly activated during imagined reaching compared to baseline. Instead, it is possible that during actual reaching, activations in the angular gyrus decreased relative to baseline.

\section{Observed versus imagined reaching}

The only difference in activation between observed and imagined reaching was in the superior occipital gyrus and in the middle occipital gyrus $(\mathrm{MT}+)$, with greater activations for observed reaching $(p<0.005$, corrected) (Fig. 6). This was expected, as there was visual movement during observation of reaching due to the moving hand, whereas there was no visual movement during imagined reaching. This difference was bilateral.

\section{Discussion}

We used fMRI to compare human cortical activations for executed, observed, and imagined reaching with the goal of identifying a mirror neuron system that represents reaching and reachingrelated behaviors. Our results suggest that such a mirror neuron system exists, and that it bears both differences and similarities to the mirror neuron system underlying grasping movements and ob- 


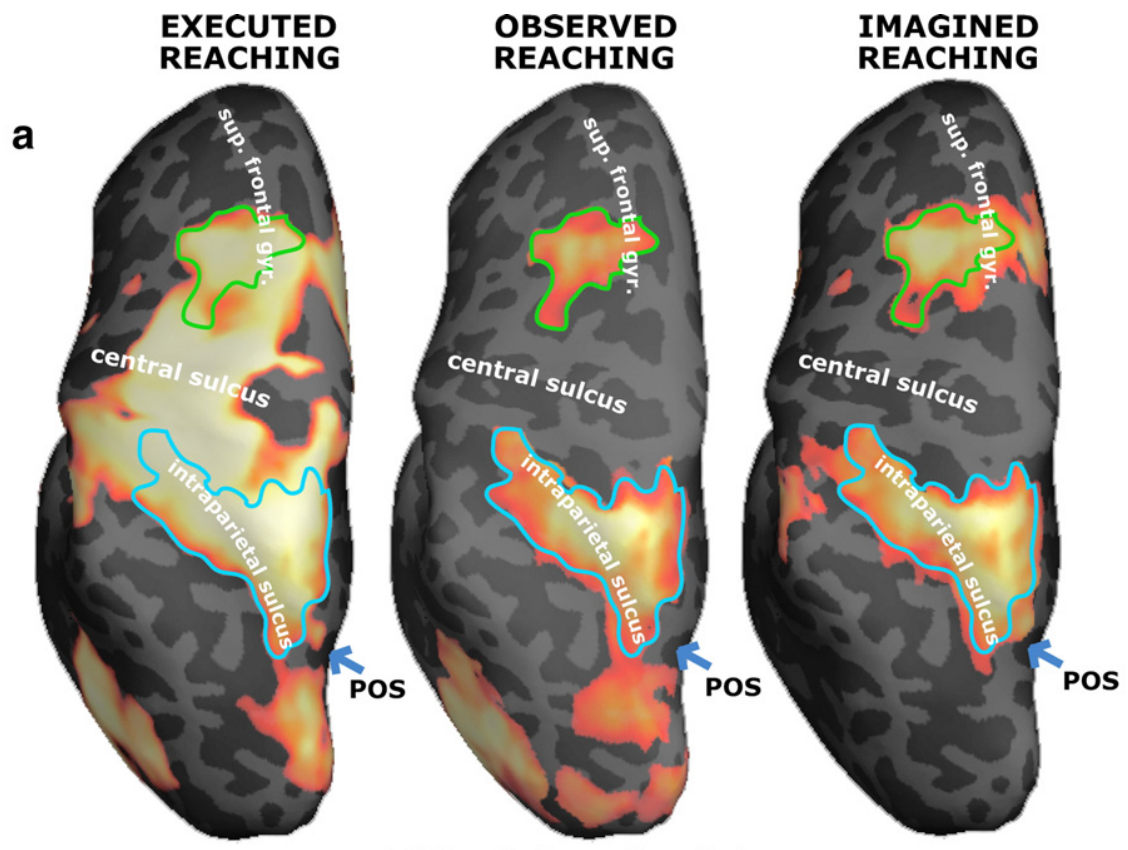

left hemisphere, dorsal view

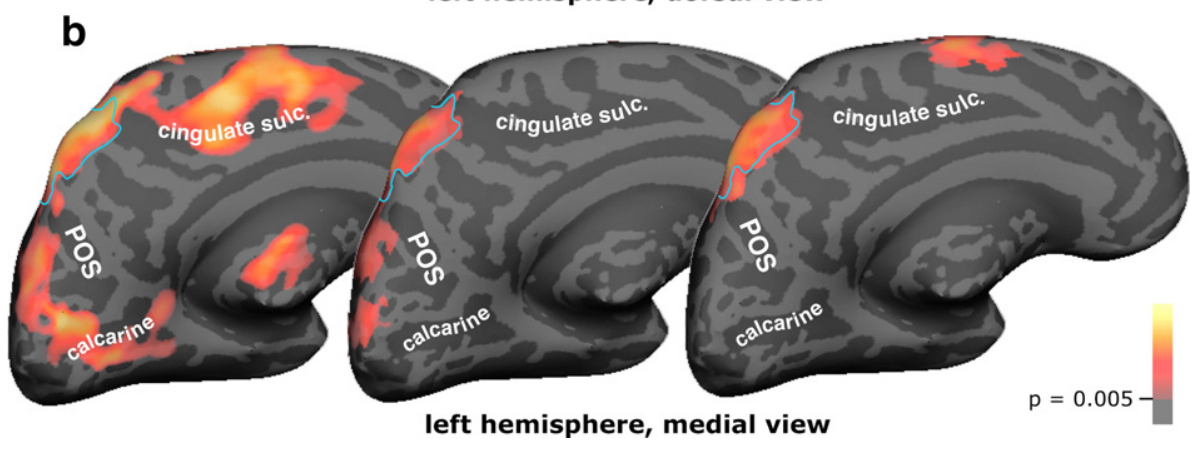

Fig. 4. Outline of overlap between executed, observed, and imagined reaching in left dorsal premotor (superior frontal sulcus and gyrus) and left posterior parietal areas, on group surface-averaged activations from 15 subjects, displayed on one subject's inflated hemisphere. The overlaps in premotor and parietal regions served as regions of interest in the percent signal change analysis. (a) Dorsal view of left hemisphere. (b) Medial view of left hemisphere. Executed, observed, and imagined reaching all activated a medial parietal area located in-between the parieto-occipital sulcus and the posterior end of the cingulate sulcus, outlined in light blue. Sup. frontal gyr. = superior frontal gyrus; POS= parieto-occipital sulcus; calcarine $=$ calcarine sulcus; cingulate sulc. $=$ cingulate sulcus.

ject manipulation. Specifically, mirror neurons for execution, imagery, and observation of reaching are found in dorsal premotor (superior frontal gyrus/sulcus) and superior parietal/intraparietal as well as in more medial parietal cortical areas. Observation of reaching without grasping does not activate Broca's area or the inferior parietal lobule (i.e. areas below the IPS, such as the supramarginal gyrus), although both executed and imagined reaching do (to a lesser extent compared to more dorsal areas). The dorsal premotor and posterior parietal activations we report here for executed

Table 4

Average MNI-space coordinates for overlaps between executed, observed, and imagined reaching activations (left hemisphere)

\begin{tabular}{llrll}
\hline Brain area & $x$ & $y$ & $z$ & $\begin{array}{l}\text { No. of } \\
\text { subjects }\end{array}$ \\
\hline Superior frontal sulcus/gyrus & -22 & -2 & 62 & 14 \\
Superior parietal gyrus (P1) & -24 & -61 & 60 & 14 \\
Intraparietal sulcus (IPS) & -30 & -49 & 48 & 13 \\
\hline
\end{tabular}

reaching are consistent with previous studies of execution of reaching-to-point or reaching-to-grasp movements as well as of pointing movements (Culham et al., 2006; Culham et al., in press; Medendorp et al., 2005; Frey et al., 2005; Grafton et al., 1996b; Astafiev et al., 2003, Prado et al., 2005).

The dorsal premotor and superior parietal/intraparietal overlap between activations for execution, observation, and imagery of reaching reported here is consistent with a fronto-parietal mirror neuron system for hand actions. Specifically, our results extend previous mirror neuron findings to visually-guided reaching movements in which an object is not grasped, touched, or manipulated. Although grasping activations and reaching activations do overlap, it appears that during reaching, as well as during imagined and observed reaching movements, premotor and parietal activations are more dorsal/superior and more medial, consistent with macaque data on fronto-parietal networks for reaching (Matelli and Luppino, 2001).

In the macaque brain, areas F2 and F7 form the dorsal premotor cortex (PMd). Area F2 is located anterior to primary motor cortex 
a Timecourse of parietal activation

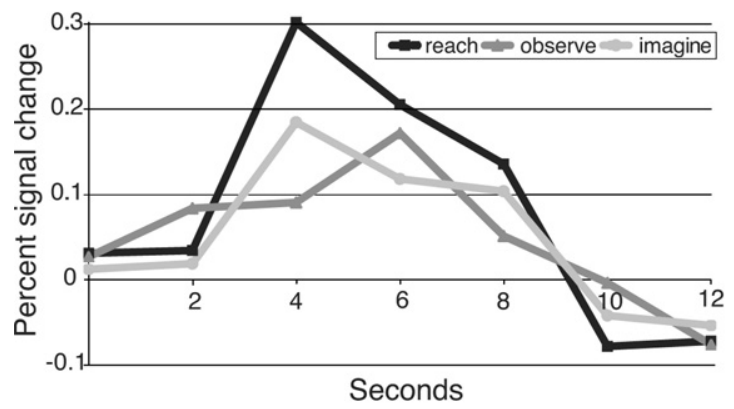

C

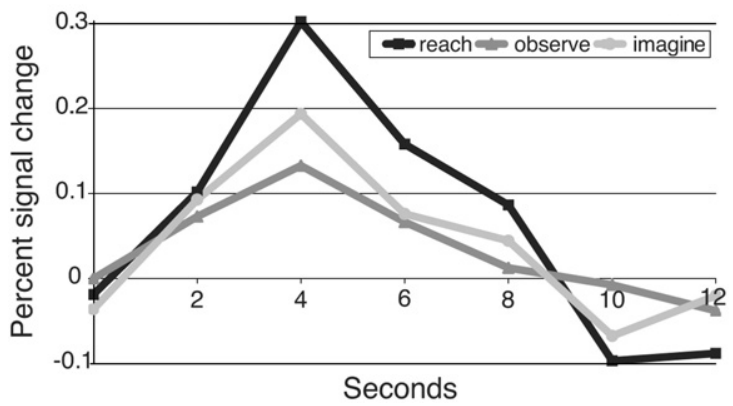

b Parietal percent signal change by condition

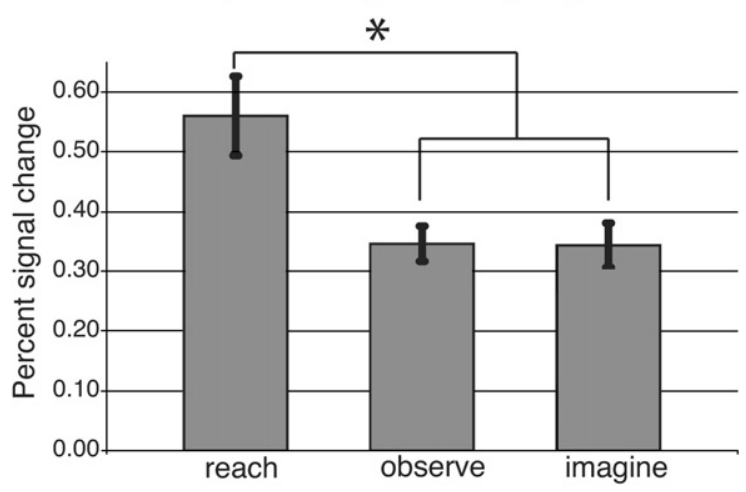

d Premotor percent signal change by condition

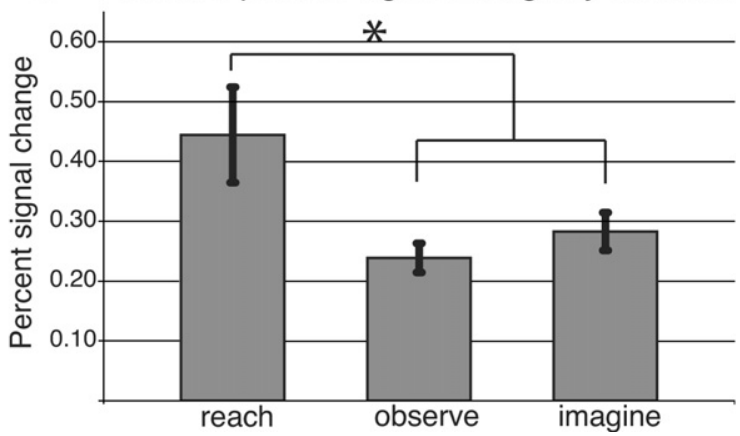

Fig. 5. Time course and percent signal change for parietal and premotor regions of interest (ROIs). (a) Parietal time course of BOLD signal change during executed, observed, and imagined reaching, versus baseline, averaged over 15 subjects. (b) Average parietal percent signal change magnitude for reaching, observed reaching, and imagined reaching, over 15 subjects. Error bars represent standard error of the mean $\left({ }^{*} p<0.05\right)$. (c) Average premotor BOLD time course, as in (a), over 15 subjects. (d) Average premotor percent signal change magnitude over 15 subjects, for executed, observed, and imagined reaching, as in (b). Reaching activations were stronger than observed and imagined reaching activations in both parietal and premotor ROIs.

and contains an arm representation. Within area F2, F2vr contains visually-responsive neurons and is a major target of parietal areas MIP and V6a, both of which contain reach-related bimodal (visual and motor) neurons. The MIP/V6a-F2vr circuit thus represents visually-guided reaching, in particular the transport phase of the hand towards the target (Matelli and Luppino, 2001). By contrast, grasping is represented by connections between the anterior intraparietal area (AIP, an area lateral and rostral in the intraparietal sulcus) and ventral premotor areas F4 and F5 in the macaque. This circuit is more lateral than the reaching circuit. A similar functional specialization of parieto-frontal circuits has recently been found in humans too (see Culham and Kanwisher, 2001; Culham et al., 2006; Culham and Valyear, 2006). Our fMRI results reported here are consistent with macaque fronto-parietal circuits for reaching, as well as with recent human neuroimaging studies on reaching and pointing. We suggest that the dorsal premotor activations obtained during executed, observed, and imagined reaching in the present study are the human homologue of macaque F2vr, and that the parietal activations we see in these three conditions include the human homologue of MIP and V6a.

Our results are consistent with a "direct-matching hypothesis" which states that actions are recognized during action observation by activating one's own motor representation of those actions (Buccino et al., 2004a). Our findings extend this matching system to imagery of action, and demonstrate that the matching system involved depends on the particular hand action, in our case, reaching. Although there is an overlap between motor substrates for grasping and reaching, there are also differences between their respective neural representations. The results presented here suggest that the main difference is in dorsal premotor and superior as well as more medial parietal activations, with greater emphasis on more medial/ superior areas for reach-related tasks than for object manipulationrelated tasks that do not involve a hand transport phase.

Superior versus inferior frontal gyrus activation during observed reaching

Whereas inferior frontal gyrus activation was present during executed and imagined reaching compared to baseline, observation of reaching versus baseline did not activate the inferior frontal gyrus, but instead only the superior frontal gyrus/sulcus. It is possible that during executed and imagined reaching, subjects may have had the intention to grasp the objects towards which they were reaching or imagining reaching. In fact, a few subjects reported reaching with the intention to grasp (although the object images were 2-dimensional). By contrast, during observation of reaching such an intention was not as obvious because the videotaped hand always stopped before getting to the point of grasping; i.e. only the transport phase was perceived. Hence in executed and imagined reaching, inferior frontal activation (Broca's area) was present, whereas observation of just the transport phase of the hand during observed reaching failed to activate the inferior frontal gyrus. This lack of inferior frontal gyrus activation is consistent with JohnsonFrey et al. (2003), who found that observation of grasping an object, 


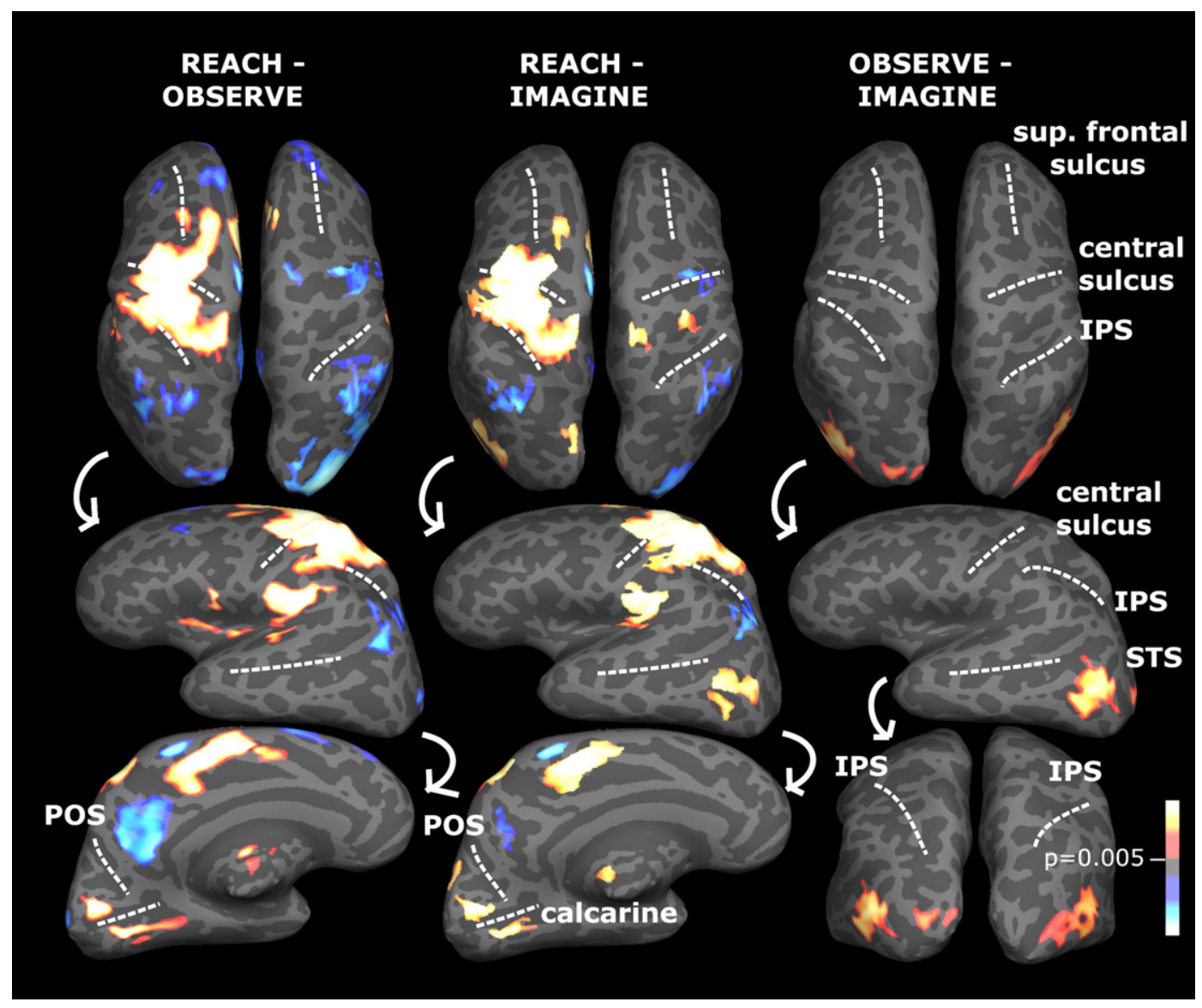

Fig. 6. Comparisons between executed and observed reaching, executed and imagined reaching, and observed versus imagined reaching. Activations are group surface-averages from 15 subjects displayed on a single subject's inflated left and right hemispheres, $p<0.005$ (corrected). Red to bright yellow codes for greater activation for the condition subtracted from. Blue activations represent greater activation for the subtracted condition. Note the lack of difference in premotor and parietal cortex for the observed versus imagined reaching comparison, suggesting that parietal and premotor mirror neurons participate equally in observation and imagery of reaching. IPS=intraparietal sulcus; calcarine=calcarine sulcus; sup. frontal sulcus=superior frontal sulcus; POS=parieto-occipital sulcus; $\mathrm{STS}=$ superior temporal sulcus.

but not observation of touching the object with the side of the palm (i.e. not with the fingers) activates the inferior frontal gyrus. In all three tasks in our study, the main component was reaching, rather than a putative intention to grasp-hence the strong dorsal premotor activations obtained here. Even if subjects may have imagined grasping at the end of the executed and imagined reach, the small extent of Broca's area activation for executed and imagined reaching compared to the more dorsal premotor activations suggests such an effect was small. The dorsal premotor activations reported here are consistent with activations reported by Culham et al. (2003) for visually-guided reaching as well as reaching-to-grasp, and with dorsal premotor activations for pointing (Medendorp et al., 2005; Astafiev et al., 2003, Hagler et al., 2007).

Our results suggest that in general, ventral premotor areas (including inferior frontal gyrus, middle frontal gyrus) are activated primarily during observation of complex hand movements that involve either pantomimed preshaping of the hand or other local interactions with an object (Grafton et al., 1996a; Buccino et al., $2001,2004 b$ ). If the task is just to observe a hand moving from one location to another as in reaching, more superior or dorsal premotor areas are also activated, such as the superior frontal gyrus, which we see activated in our task.

\section{Superior versus inferior parietal activations}

Likewise, in parietal cortex, observation of hand-object interactions activates more inferior parietal areas, such as the parietal operculum, whereas observation of reaching without grasping activates superior parietal areas, such as the superior parietal gyrus (P1) within the superior parietal lobule, in addition to the IPS. Although executed reaching activated area AIP in our study (defined as the junction between the intraparietal sulcus and the postcentral sulcus, see Culham et al., 2006; Frey et al., 2005), our activations also extended more medially into the superior aspect of the precuneus compared to grasping-related activations reported by most studies (Grèzes et al., 2003; Rizzolatti et al., 1996b) or activations for local finger actions such as playing the guitar or imitating finger lifting (Buccino et al., 2004b; Iacoboni et al., 1999). This pattern was true for observation of reaching and imagery of reaching as well, suggesting a more dorsal and medial mirror neuron system for reaching-related tasks than for object manipulation tasks. An interesting exception is found by Binkofski et al. (1999), who did find superior parietal activations during complex object manipulation in the dark, in addition to inferior frontal and inferior parietal activations (including AIP). However, the activations depended on the exploration 
conditions. When complex objects that were not easily recognized were explored, both left and right superior parietal lobules were activated compared to rest. When more easily recognizable objects were explored, where naming of the objects was possible, the left superior parietal lobule was not activated, whereas the right superior parietal lobule was. Binkofski et al. suggest that the superior parietal lobule is involved in proprioception and that there is a hand/finger representation in the superior parietal lobule. The fact that easily recognizable object manipulation did not activate the left superior parietal lobule suggests that exploration of objects with more complex spatial characteristics will activate this region. This spatial aspect may be important during reaching too, e.g. during the visuomotor guidance of the arm to the appropriate location. It is possible that the superior parietal lobule is involved in multiple motor representations, and not exclusively in reaching. Note that as Binkofski et al. (1999) only investigated the execution aspect of complex object manipulation, it is not known if observation of the same movements would also have activated superior parietal areas. The important finding in our paper, however, is that mirror neurons for executed, observed, and imagined reaching are located in superior and medial parietal areas, which is consistent with parietofrontal circuits for reaching in both macaques and humans. The overlap between all three conditions was not found in Broca's area, where mirror neurons for object manipulation are found.

While observed and imagined reaching per se have not been studied previously, the superior and medial parietal activations obtained here for execution of reaching are consistent with previous neuroimaging studies of execution tasks similar to reaching. Connolly et al. (2003) and Astafiev et al. (2003) propose that the human equivalent of MIP and V6A, also called the parietal reach region (PRR), is located medial to the IPS and on the medial wall of the parietal lobe (precuneus), based on pointing-related fMRI activations in the human brain. The precuneus and medial IPS/ superior parietal activations reported here are consistent with their proposed location. Similar parietal activations were also found by Medendorp et al. (2005), Desmurget et al. (2001), Hagler et al. (2007), Culham et al. (in press) and Prado et al. (2005). Culham et al. (in press) suggest that the superior parieto-occipital cortex, broadly consistent with our activations in-between the parietooccipital sulcus (POS) and the posterior end of the cingulate sulcus (Fig. 4b), may be especially important for the transport phase of the reaching movement. In our study, all three conditions activated areas immediately anterior to the POS, whereas executed and observed, but not imagined reaching, activated presumably more visual areas posterior to the POS. The activation anterior to the POS is consistent with the proposal that the human homologue of macaque V6A is anterior to the POS (Culham et al., in press), which overlaps with what Connolly et al. (2003) call the parietal reach region (PRR). If Culham et al.'s interpretation of this area is correct, our results suggest that this area contains mirror neurons for the executed, observed, and imagined transport phase of reaching. Fig. 6 shows that this area was equally activated during observed and imagined reaching, and that executed reaching activated more superior aspects of this area more strongly than either observed or imagined reaching.

Prado et al. (2005) argue for a dissociation between brain circuits involved in reaching toward central versus peripheral visual targets. Whereas in their study the medial IPS was active in both, activation centered on the parieto-occipital junction (POJ) on the medial wall was present only during reaching to peripheral targets. Our activations for reaching are in agreement with Prado et al.'s (2005) activations for reaching to peripheral targets. In our study, targets were presented in the periphery, and no saccade was executed towards targets prior to executed, observed or imagined reaching. All three conditions activated an area located in-between the POS and the posterior end of the cingulate sulcus, with reaching and observed reaching extending posterior to the POS as well (see Fig. 4b). While we did not compare reaching towards central and peripheral targets, we can conclude that there are mirror neurons for observed, imagined, and executed reaching to peripheral targets around and slightly anterior to the parietooccipital junction, consistent with Prado et al.'s (2005) results for reaching to peripheral targets.

\section{Dorsal premotor cortex and eye movements}

Although we monitored subjects' eye movements outside the scanner and confirmed that they maintained fixation, it could be argued that some of the premotor activations reported here may in fact be eye movement-related activations rather than mirror neuron activations for reaching. Several aspects of our results speak against this argument. Firstly, superior frontal gyrus/sulcus activation was absent or very weak in the right hemisphere during observed and imagined reaching (Fig. 2). If indeed subjects had moved their eyes substantially during all three tasks, one would expect bilateral and more symmetric activations in dorsal premotor cortex, which we did not find. Secondly, the proposed location of the frontal eye fields (FEF) in humans is at the junction of the precentral and superior frontal sulci (Koyama et al., 2004; Culham et al., 1998), whereas our dorsal premotor focus of activation for observed and imagined reaching was anterior to this junction (see Figs. 2 and 4). The dorsal premotor activation reported here was located partly on the superior frontal gyrus and inside the superior frontal sulcus anterior to the precentral sulcus. According to Blanke et al. (2000), putative human FEF even overlaps with the precentral gyrus, which is well posterior to the proposed mirror neuron activation reported here. Both Koyama et al. and Culham et al. report a second area related to eye movements in the inferior precentral sulcus. In our study, observation of reaching elicited no such inferior precentral sulcus activations, whereas imagined reaching yielded a very small activation in the inferior precentral sulcus (Fig. 2). These findings suggest that the overlap in activations for observed, imagined, and executed reaching reported here is not likely due to eye movements during each of these tasks, even if some eye movement may have occurred. Culham et al. (2003) obtained activations in the FEF during visually-guided reaching and grasping, which they suggested may be due to a subpopulation of neurons in FEF that is activated during arm movements. This would support our hypothesis that part of dorsal premotor cortex in humans represents arm movements. It is possible that some of the dorsal premotor activations reported here partially overlap with such a population of FEF neurons, although as stated above our activations are mainly anterior to the FEF. A similar anterior focus was also obtained by Astafiev et al. (2003) for pointing, consistent with our results.

In conclusion, we have demonstrated the existence of a frontoparietal mirror neuron system for executed, observed, and imagined reaching. Whereas grasping has been studied before, very few fMRI studies have investigated reach-related mirror neuron activations, perhaps due to the difficulty with larger hand movements that involve a transport phase. Our results suggest that reach-related mirror neuron activations are present in the superior frontal gyrus and sulcus, as well as in intraparietal and superior/medial parietal 
areas. Reaching drives both areas more than observation and imagery of reaching, whereas activations for observation and imagery of reaching in these two regions are equally strong. The present results help shed light on the complex mosaic of visuo-motor representations in the human brain, and extend previous studies of mirror neuron activations in humans to reaching.

\section{Acknowledgments}

This work was funded by NSF BCS 0224321 to M.I.S. and by NIH 5T32MH020002, which funded J.D.N. The authors are grateful to Emanuel Todorov and Ruey-Song Huang for helpful input, and to Alan E. Robinson and two anonymous reviewers for comments on an earlier draft. We would also like to thank the UCSD Center for Functional Magnetic Resonance Imaging, and in particular Giedrius T. Buracas and Larry May, for support and technical assistance.

\section{References}

Andersen, R.A., Buneo, C.A., 2002. Intentional maps in posterior parietal cortex. Annu. Rev. Neurosci. 25, 189-220.

Astafiev, S.V., Shulman, G.L., Stanley, C.M., Snyder, A.Z., Van Essen, D.C., Corbetta, M., 2003. Functional organization of human intraparietal and frontal cortex for attending, looking, and pointing. J. Neurosci. 23 (11), 4689-4699.

Astafiev, S.V., Stanley, C.M., Shulman, G.L., Corbetta, M., 2004. Extrastriate body area in human occipital cortex responds to the performance of motor actions. Nat. Neurosci. 7 (5), 542-548.

Battaglia-Mayer, A., Ferraina, S., Mitsuda, T., Marconi, B., Genovesio, A., Onorati, P., et al., 2000. Early coding of reaching in parietooccipital cortex. J. Neurophysiol. 83 (4), 2374-2391.

Binkofski, F., Buccino, G., Posse, S., Seitz, R.J., Rizzolatti, G., Freund, H.-J., 1999. A fronto-parietal circuit for object manipulation in man: evidence from an fMRI study. Eur. J. Neurosci. 11, 3276-3286.

Blanke, O., Spinelli, L., Thut, G., Michel, C.M., Perrig, S., Landis, T., Seeck, M., 2000. Location of the human frontal eye field as defined by electrical cortical stimulation: anatomical, functional and electrophysiological characteristics. NeuroReport 11 (9), 1907-1913.

Buccino, G., Binkofski, F., Fink, G.R., Fadiga, L., Fogassi, L., Gallese, V., Seitz, R.J., Zilles, K., Rizzolatti, G., Freund, H.-J., 2001. Action observation activates premotor and parietal areas in a somatotopic manner: an fMRI study. Eur. J. Neurosci. 13, 400-404.

Buccino, G., Binkofski, F., Riggio, L., 2004a. The mirror neuron system and action recognition. Brain Lang. 89 (2), 362-369.

Buccino, G., Vogt, S., Ritzl, A., Fink, G.R., Zilles, K., Freund, H.-J., Rizzolatti, G., 2004b. Neural circuits underlying imitation learning of hand actions: an event-related fMRI study. Neuron 42, 323-334.

Buneo, C.A., Jarvis, M.R., Batista, A.P., Andersen, R.A., 2002. Direct visuomotor transformations for reaching. Nature 416 (6881), 632-636.

Chapman, H., Gavrilescu, M., Wang, H., Kean, M., Egan, G., Castiello, U., 2002. Posterior parietal cortex control of reach-to-grasp movements in humans. Eur. J. Neurosci. 15 (12), 2037-2042.

Connolly, J.D., Andersen, R.A., Goodale, M.A., 2003. FMRI evidence for a 'parietal reach region' in the human brain. Exp. Brain Res. 153 (2), $140-145$.

Cox, R.W., 1996. AFNI: software for analysis and visualization of functional magnetic neuroimages. Comput. Biomed. Res. 29, 162-173.

Culham, J.C., Kanwisher, N.G., 2001. Neuroimaging of cognitive functions in human parietal cortex. Curr. Opin. Neurobiol. 11 (2), 157-163.

Culham, J.C., Valyear, K.F., 2006. Human parietal cortex in action. Curr. Opin. Neurobiol. 16, 205-212.

Culham, J.C., Brandt, S.A., Cavanagh, P., Kanwisher, N.G., Dale, A.M.,
Tootell, R.H., 1998. Cortical fMRI activation produced by attentive tracking of moving targets. J. Neurophysiol. 80, 2657-2670.

Culham, J.C., Danckert, S.L., DeSouza, J.F.X., Gati, J.S., Menon, R.S., Goodale, M.A., 2003. Visually guided grasping produces PMRI activation in dorsal but not ventral stream areas. Exp. Brain Res. 153, $180-189$.

Culham, J.C., Cavina-Pratesi, C., Singhal, A., 2006. The role of parietal cortex in visuomotor control: what have we learned from neuroimaging? Neuropsychologia 44, 2668-2684.

Culham, J.C., Gallivan, J., Cavina-Pratesi, C., and Quinlan, D.J., in press. FMRI investigations of reaching in ego space in human superior parietooccipital cortex. In: Klatzky, R., McWhinney, B., Behrmann, M. (Eds.), Embodiment, Ego-Space, and Action. Erlbaum, Madwah NJ.

Dale, A.M., Fischl, B., Serreno, M.I., 1999. Cortical surface-based analysis I: segmentation and surface reconstruction. NeuroImage 9, 179-194.

Decety, J., Grèzes, J., 1999. Neural mechanisms subserving the perception of human actions. Trends Cogn. Sci. 3 (5), 172-178.

Decety, J., Grèzes, J., Costes, N., Perani, D., Jeannerod, M., Procyk, E., Grassi, F., Fazio, F., 1997. Brain activity during observation of actions. Influence of action content and subject's strategy. Brain 120, 1763-1777.

Desmurget, M., Gréa, H., Grethe, J.S., Prablanc, C., Alexander, G.E., Grafton, S.T., 2001. Functional anatomy of nonvisual feedback loops during reaching: a positron emission tomography study. J. Neurosci. 21 (8), 2919-2929.

DeSouza, J.F., Dukelow, S.P., Gati, J.S., Menon, R.S., Andersen, R.A., Vilis, T., 2000. Eye position signal modulates a human parietal pointing region during memory-guided movements. J. Neurosci. 21 (8), 2919-2928.

Duvernoy, 1999. Human Brain Atlas, 2nd edition. Springer-Verlag, Wien.

Fattori, P., Gamberini, M., Kutz, D.F., Galletti, C., 2001. 'Arm-reaching' neurons in the parietal area V6A of the macaque monkey. Eur. J. Neurosci. 13 (12), 2309-2313.

Ferraina, S., Battaglia-Mayer, A., Genovesio, A., Marconi, B., Onorati, P., Caminiti, R., 2001. Early coding of visuomanual coordination during reaching in parietal area PEc. J. Neurophysiol. 85 (1), 462-467.

Fischl, B., Sereno, M.I., Dale, A.M., 1999a. Cortical surface-based analysis II: inflation, flattening, and a surface-based coordinate system. NeuroImage 9, 195-207.

Fischl, B., Sereno, M.I., Tootell, R.B., Dale, A.M., 1999b. High-resolution intersubject averaging and a coordinate system for the cortical surface. Hum. Brain Mapp. 8, 272-284.

Fogassi, L., Gallese, V., Fadiga, L., Rizzolatti, G., 1998. Neurons responding to the sight of goal-directed hand/arm actions in the parietal area PF (7b) of the macaque monkey. Abstr.-Soc. Neurosci. 24, 257.5.

Frey, S.H., Vinton, D., Norlund, R., Grafton, S.T., 2005. Cortical topography of human anterior intraparietal cortex active during visually guided grasping. Cogn. Brain Res. 23, 397-405.

Galletti, C., Fattori, P., Kutz, D.F., Battaglini, P.P., 1997. Arm movementrelated neurons in the visual area V6A of the macaque superior parietal lobule. Eur. J. Neurosci. 9 (2), 410-413.

Grafton, S.T., Arbib, M.A., Fadiga, L., Rizzolatti, G., 1996a. Localization of grasp representation in humans by positron emission tomography. 2: Observation compared with imagination. Exp. Brain Res. 112, 103-111.

Grafton, S.T., Fagg, A.H., Woods, R.P., Arbib, M.A., 1996b. Functional anatomy of pointing and grasping in humans. Cereb. Cortex 6 (2), 226-237.

Grefkes, C., Ritzl, A., Zilles, K., Fink, G.R., 2004. Human medial intraparietal cortex subserves visuomotor coordinate transformations. NeuroImage 23 (4), 1494-1506.

Grèzes, J., Decety, J., 2001. Functional anatomy of execution, mental simulation, observation, and verb generation of actions: a meta-analysis. Hum. Brain Mapp. 12, 1-19.

Grèzes, J., Armony, J.L., Rove, J., Passingham, R.E., 2003. Activations related to "mirror" and "canonical" neurones in the human brain: an fMRI study. NeuroImage 18, 928-937.

Hagler, D.J., Saygin, P.A., Sereno, M.I., 2006. Smoothing and cluster thresholding for cortical surface-based group analysis of fMRI data. NeuroImage 33, 1093-1103. 
Hagler Jr., D.J., Riecke, L., Sereno, M.I., 2007. Parietal and superior frontal visuospatial maps activated by pointing and saccades. NeuroImage 35 (4), 1562-1577.

Hamzei, F., Rijntjes, M., Dettmers, C., Glauche, V., Weiller, C., Buchel, C., 2003. The human action recognition system and its relationship to Broca's area: an fMRI study. NeuroImage 19, 637-644.

Iacoboni, M., Woods, R.P., Brass, M., Bekkering, H., Mazziotta, J.C., Rizzolatti, G., 1999. Cortical mechanisms of human imitation. Science 286, 2526-2528.

Johnson, P.B., Ferraina, S., Bianchi, L., Caminiti, R., 1996. Cortical networks for visual reaching: physiological and anatomical organization of frontal and parietal lobe arm regions. Cereb. Cortex 6 (2), 102-119.

Johnson-Frey, S.H., Maloof, F.R., Newman-Norlund, R., Farrer, C., Inati, S., Grafton, S.T., 2003. Actions or hand-object interactions? Human inferior frontal cortex and action observation. Neuron 39, 1053-1058.

Johnson-Frey, S.H., Newman-Norlund, R.N., Grafton, S.T., 2005. A distributed left hemisphere network active during planning of everyday tool use skills. Cereb. Cortex 15, 681-695.

Kalaska, J.F., 1996. Parietal cortex area 5 and visuomotor behavior. Can. J. Physiol. Pharmacol. 74, 483-498.

Kawashima, R., Naitoh, E., Matsumura, M., Itoh, H., Ono, S., Satoh, K., et al., 1996. Topographic representation in human intraparietal sulcus of reaching and saccade. NeuroReport 7, 1253-1256.

Koyama, M., Hasegawa, I., Osada, T., Adachi, Y., Nakahara, K., Miyashita, Y., 2004. Functional magnetic resonance imaging of macaque monkeys performing visually guided saccade tasks: comparison of cortical eye fields with humans. Neuron 41, 795-807.

Króliczak, G., Cavina-Pratesi, C., Goodman, D.A., Culham, J.C., 2007. What does the brain do when you fake it? An fMRI study of pantomimed and real grasping. J. Neurophysiol. 97, 2410-2422.

Lacquaniti, F., Perani, D., Guigon, E., Bettinardi, V., Carrozzo, M., Grassi, F., et al., 1997. Visuomotor transformations to memorized targets: a PET study. NeuroImage 5, 129-146.

Matelli, M., Luppino, G., 2001. Parietofrontal circuits for action and space perception in the macaque monkey. NeuroImage 14, S27-S32.

Medendorp, W.P., Goltz, H.C., Crawford, J.D., Vilis, T., 2005. Integration of target and effector information in human posterior parietal cortex for the planning of action. J. Neurophysiol. 93 (2), 954-962.

Molnar-Szakacs, I., Kaplan, J., Greenfield, P., Iacoboni, M., 2006. Ob- serving complex action sequences: the role of the fronto-parietal mirror neuron system. NeuroImage 33, 923-935.

Muakkassa, K.F., Strick, P.L., 1979. Frontal lobe inputs to primate motor cortex: evidence for four somatotopically organized 'premotor' areas. Brain Res. 177, 176-182.

Nishitani, N., Hari, R., 2000. Temporal dynamics of cortical representation for action. Proc. Natl. Acad. Sci. 97 (2), 913-918.

Perrett, D.I., Harries, M.H., Bevan, R., Thomas, S., Benson, B., Mistlin, A.J., Chitty, A.J., Hietanen, J.K., Ortega, J.E., 1989. Frameworks of analysis for the neural representation of animate objects and actions. J. Exp. Biol. 146, 87-113.

Prado, J., Clavagnier, S., Otzenberger, H., Scheiber, C., Kennedy, H., Perenin, M.-T., 2005. Two cortical systems for reaching in central and peripheral vision. Neuron 48, 849-858.

Preuss, T.M., Stepniewska, I., Kaas, J.H., 1996. Movement representation in the dorsal and ventral premotor areas of owl monkeys: a microstimulation study. J. Comp. Neurol. 371, 649-676.

Rizzolatti, G., Craighero, L., 2004. The mirror-neuron system. Annu. Rev. Neurosci. 27, 169-192.

Rizzolatti, G., Camarda, R., Fogassi, L., Gentilucci, M., Luppino, G., Matelli, M., 1988. Functional organization of inferior area 6 in the macaque monkey. Exp. Brain Res. 71, 491-507.

Rizzolatti, G., Fadiga, L., Gallese, V., Fogassi, L., 1996a. Premotor cortex and the recognition of motor actions. Cogn. Brain Res. 3, 131-141.

Rizzolatti, G., Fadiga, L., Matelli, M., Bettinardi, V., Paulesu, E., Perani, D., Fazio, F., 1996b. Localization of grasp representations in humans by PET: 1. Observation versus execution. Exp. Brain Res. 111, 246-252.

Stepniewska, I., Fang, P.-C., Kaas, J.H., 2005. Microstimulation reveals specialized subregions for different complex movements in the posterior parietal cortex of prosimian primates. Proc. Natl. Acad. Sci. 102, 4878-4883.

Tai, Y.F., Scherfler, C., Brooks, D.J., Sawamoto, N., Castiello, U., 2004. The human premotor cortex is "mirror" only for biological actions. Curr. Biol. 14, 117-120.

Ward, B.D., 2000a. Deconvolution analysis of fMRI time series data. AFNI $3 \mathrm{dDeconvolve}$ documentation, Medical College of Wisconsin.

Ward, B.D., 2000b. Simultaneous inference for fMRI data. AFNI $3 \mathrm{dDeconvolve}$ documentation, Medical College of Wisconsin. 